\title{
Empyema necessitans, a rare complication of empyema thoracis
}

\section{Introduction}

Empyema is defined as a collection of pus in the pleural space. When the pus extends through the parietal pleura into adjacent tissue, it is termed as empyema necessitans.

\section{Case presentation}

A 18 year old male with body mass index (BMI) 15.5 presented with pain and swelling in his right posterior chest wall for 4 months. $\mathrm{He}$ had subjective fever which got subsided for last 15 days. However, the swelling didn't decrease size in size over course of time. Chest X-ray posterior anterior and right lateral view revealed no abnormality. His erythrocyte sedimentation rate (ESR) was 27 millimeter in $1^{\text {st }}$ hour. White blood cell was 11,000 per microliter, neutrophil was $68 \%$ and lymphocyte $27 \%$. Since, the swelling was confined to soft tissue, lobulated in outline and boggy in palpation, a provisional diagnosis of hemangioma was made. However, no local rise in temperature was noted. Since, the swelling was confined to soft tissue, contrast enhanced Magnetic Resonance Imaging (MRI) was advised which showed a peripheral rim enhancing, large, multilobulated T1WI hypointense and T2WI hyperintense lesion in right posterior chest wall having multiple internal septations. The lesion was involving the intercostal space and intercostal muscles. Cortical irregularity and altered marrow signal intensity was noted at $6^{\text {th }}$ and $7^{\text {th }}$ ribs of right posterior chest wall. Similar characteristics lesion was noted in the right posterior pleural space which appeared communicating with the posterior chest wall lesion. Ultrasound (US)
Volume 5 Issue 6 - 2018

\author{
Bishwajit Bhowmik,' Mahesh Chaudhary,' \\ Anindita Datta,' Manmohan Bir Shrestha,' \\ Nazahath Abbas ${ }^{2}$ \\ 'Department of Radiology \& Imaging, Bangabandhu Sheikh Mujib \\ Medical University, Bangladesh \\ ${ }^{2}$ Yerevan State Medical University, Armenia
}

\begin{abstract}
Correspondence: Mahesh Chaudhary, Department of Radiology \& Imaging, Bangabandhu Sheikh Mujib Medical University, Dhaka, Bangladesh,

Email maheshchawdhary@hotmail.com
\end{abstract}

Received: September 23, 2018 | Published: November 21, 2018

examination of the swelling was done which showed hypoechoic, multi lobulated mass which showed no vascularity on color flow. Hence, ultrasound guided fine needle aspiration (FNA) was done; from which thick purulent yellowish colored pus was aspirated and was sent for gram stain, acid fast bacillus, culture and sensitivity. Pus culture revealed Mycobacterium tuberculosis. Based on this, thoracotomy with decortication was done. Patient was treated with intravenous antibiotics and discharged home on oral antibiotics with significant improvement (Figure $1 \&$ Figure 2).
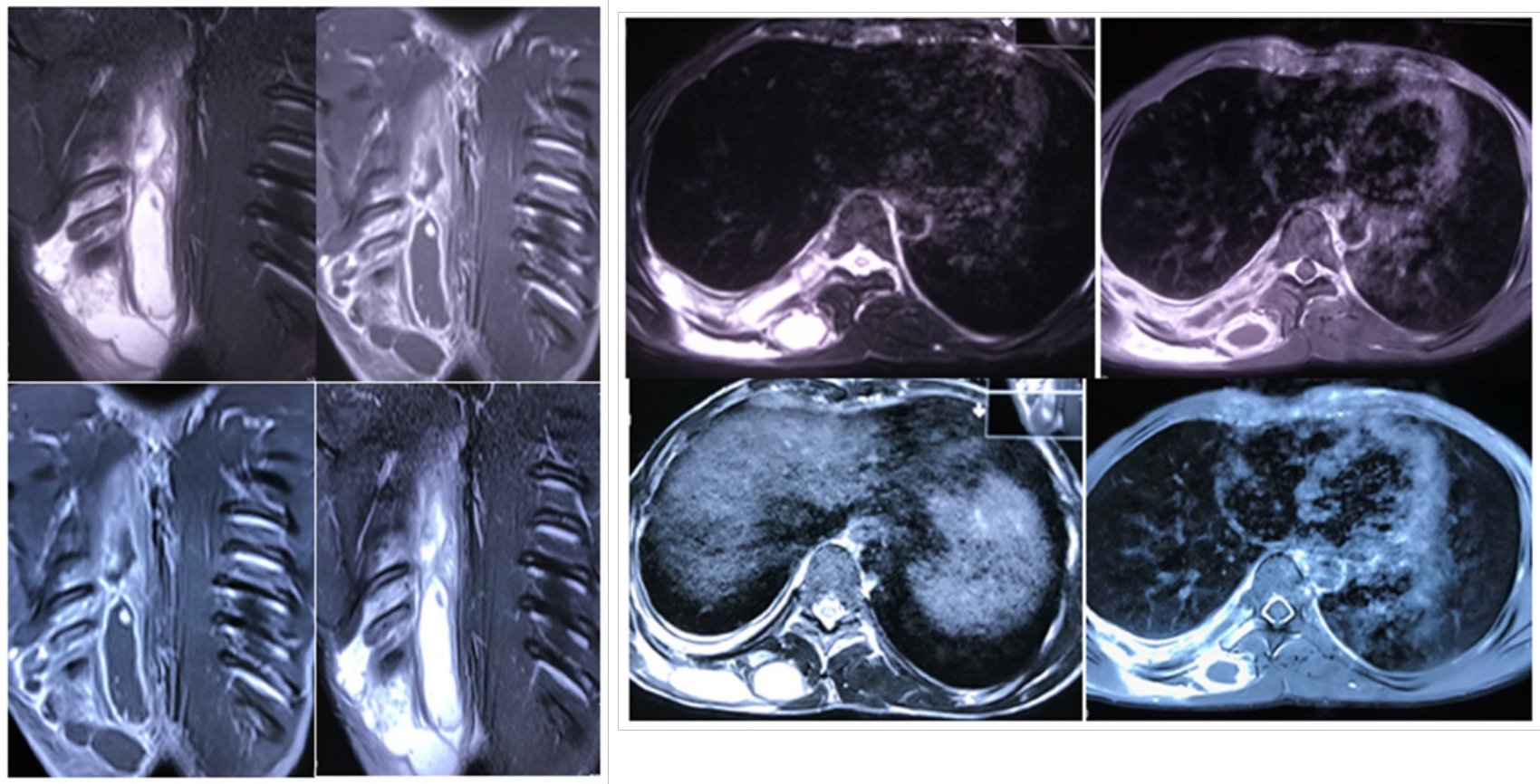

Figure I Contrast enhanced MRI of chest: Coronal and Axial TI and T2 images. 


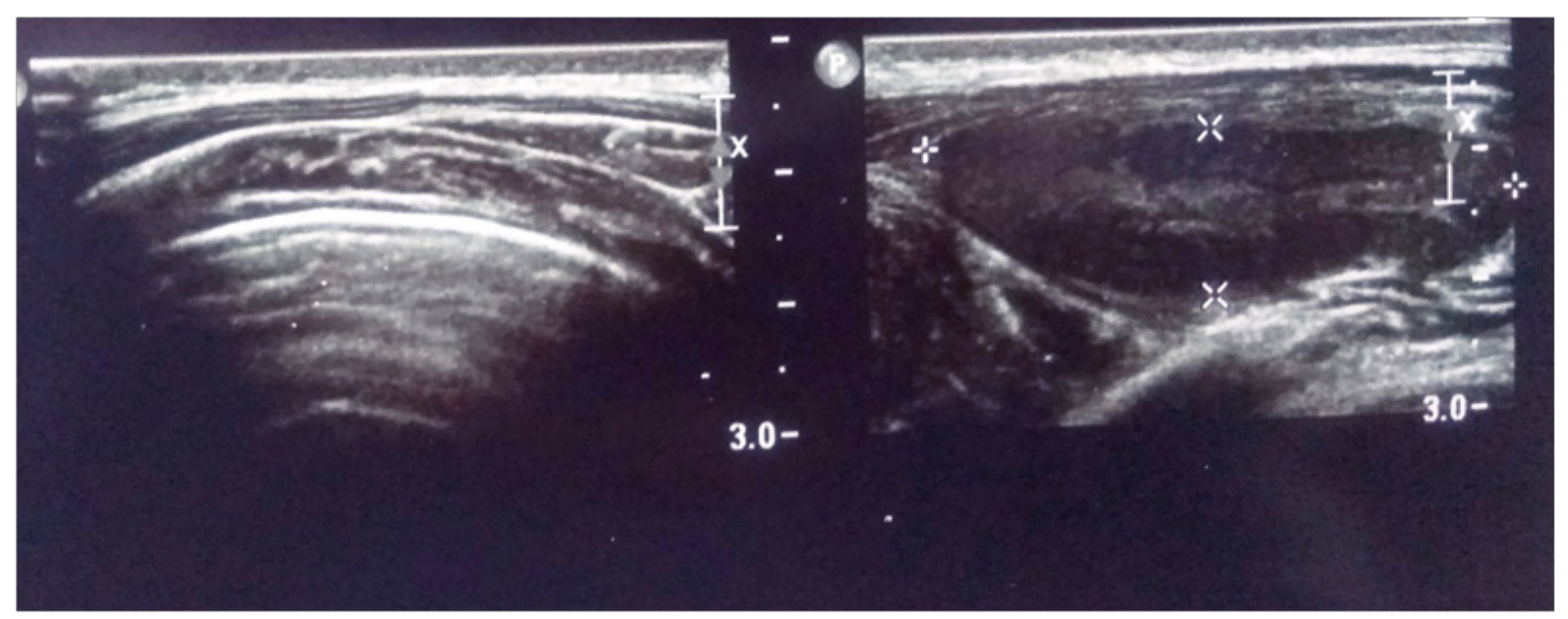

Figure 2 USG of back of chest:Transverse and Longitudinal views.

\section{Discussion}

Empyema necessitans is a rare complication in the present era after the development of antibiotics. The most common etiology being mycobacterium tuberculosis. ${ }^{2}$ The chronic nature of the infection corresponds with the slow progression of disease. The granulomatous infection creates a tract from the pleural space through parietal pleura to the surrounding soft tissues and skin of chest wall forming a fistula. ${ }^{3}$ Bronchopleural fistulas and pyopneumothorax are common complications of empyema. Other rare complications like purulent pericarditis, pulmonary abscess, peritonitis from extension through diaphragm and osteomyelitis of adjacent rib can occur. ${ }^{4}$

\section{Conclusion}

Empyema necessitans is a result of neglected or inadequately treated pulmonary infection. ${ }^{5}$ Immunocompromised states in any form not only adds to the progression of infection but also hinders in the diagnosis of disease making Monteux test negative. ${ }^{6}$ The delay in the treatment of empyema necessitans thereby increases morbidity and mortality. ${ }^{7}$ The most challenging part is to identify the disease as empyema necessitans. Early intervention by sterilizing the pleural cavity, draining the pus and adding appropriate antibiotics aids in rapid improvement, prevents recurrence and reduces morbidity and mortality associated with this disease.

\section{Acknowledgements}

None.

\section{Conflict of interest}

The authors declare that there is no conflict of interest.

\section{References}

1. Bhanja S, Guha A, Samanta A, et al. A Case of Empyema Necessitans: An Uncommon Presentation of Empyema. Int J Pediatr. 2017;5(7):53515355 .

2. Kellie SP, Shaib F, Forster D, et al. Empyema Necessitatis. Chest. 2010;138(4):39A

3. Kono SA, Nauser TD. Contemporary empyema necessitatis. The American Journal of Medicine. 2007;120(4):303-305.

4. Yauba MS, Ahmed H, Imoudu IA, et al. Empyema Necessitans Complicating Pleural Effusion Associated with Proteus Species Infection : A Diagnostic Dilemma. Case Rep Pediatric. 2015;2015:108174.

5. Tamura L, Hasoon M, Bandlamuri M, et al. Empyema Necessitans (EN): Critical Care Importance of Appropriate Pleural Drainage. Internet Scientific Publications. 2015;17(1):10-13.

6. Magness DJ. Empyema necessitans caused by Mycobacterium tuberculosis in an immunocompetent patient. WMJ. 2013;112(3):129-130. 